\title{
Alternative Solutions to Mitigate Problems due to Neutral Conductors Theft in MV (Medium Voltage) Power Distribution Systems
}

\author{
Fabio Romero ${ }^{1}$, Alden Uehara Antunes ${ }^{1}$, Fernando Locks Lange ${ }^{1}$, Dário Takahata ${ }^{1}$, André Meffe ${ }^{1}$, Carlos César \\ Barioni de Oliveira ${ }^{1}$ and Hamilton Bueno de Souza ${ }^{2}$ \\ 1. Department of Engineering Research and Development, Daimon Engineering \& Systems, São Paulo 01310200, Brazil \\ 2. Department of Engineering, AES Eletropaulo Distribution Utility, São Paulo 06460040, Brazil
}

Received: July 08, 2014 / Accepted: October 10, 2014 / Published: December 31, 2014.

\begin{abstract}
This paper aims at analyzing the impact of the neutral conductor absence at specific sections over the performance of the power distribution lines, and proposing alternative solutions to mitigate the problems caused by the neutral conductor theft. Simulations are made by the software Interplan and show that the absence of neutral conductor at specific sections of power distribution lines may increase the neutral-to-ground voltages, which compromises the system's safety. The solution developed keeps the technical performance of the power distribution system at satisfactory levels, regarding the voltage profile, or, at least, close to the level before the neutral conductor's theft.
\end{abstract}

Key words: Power distribution systems, conductor theft, system safety, neutral-to-ground voltages, grounding.

\section{Introduction}

Neutral conductor theft at specific sections of power distribution systems is responsible for many issues regarding the operation of distribution feeders, especially at rural areas and suburbs. In Brazil, this problem has been increasing due to the easiness associated with the reselling of the cable materials (copper and aluminium) at the black market. The unpredictability of this action (due to the characteristics of the loads supplied by the feeders involved) may cause undesirable consequences to power companies, such as impacts at the energy quality, equipment damages, income losses, increase of the interruption levels and step and touch potentials, and high costs in order to replace the conductors (copper or aluminium conductors).

Corresponding author: Fabio Romero, engineer, research fields: electrical power distribution planning, distribution losses, smart grid, protection of distribution lines, grounding, lightning electromagnetic fields, and power energy quality. E-mail: fabio.romero@daimon.com.br.
One of the Brazilian's distributions utility that have suffered injuries due to the absence of neutral conductors in its power distribution system is AES (Applied Energy Service) Eletropaulo. The Company is responsible for about six million customers in the State of Sao Paulo, in the Southeast of Brazil. AES Eletropaulo power distribution system is characterized by the presence of a common neutral conductor for the MV (medium voltage) and LV (low-voltage) lines, multigrounded at every $300 \mathrm{~m}$ distance and at every pole where there is an equipment installed (distribution transformers, reclosers, capacitor banks, voltage regulators, etc.). The common neutral multigrounded was employed in order to have a low impedance path back to the substation [1].

The aim of this work is to analyze the impact of neutral conductor's absence at specific sections of the line over the performance and safety of distribution network, and to propose technical solutions to mitigate the damages due to the neutral conductor theft. 
The analysis presented in this article is part of the activities of an R \& D (research and development) Project in partnership with AES Eletropaulo and the conclusions are in application in the RGR-104 feeder.

The solution developed specifies suitable $R g$ (ground resistance) values at specific grounding points along the distribution feeder as to keep the system's performance at satisfactory technical and safety levels [2].

The study was performed for the RGR-104 feeder due to the high incidence of neutral's conductor theft. As a reference to the analysis, the performance of the RGR-104 feeder was evaluated taking into account the neutral conductor at all sections.

\section{System Description}

Simulations are performed with the software Interplan [3] and refer to the RGR-104 distribution network. The RGR-104 is a $13.2 \mathrm{kV}$ feeder with $51 \mathrm{~km}$ MV line and, according to AES Eletropaulo, the neutral conductors are absent in $20 \%$ of the total length, as shown in Fig. 1.

Neutral conductors installed have the following parameters:

- main feeder: 3/0 AWG (American Wire Gauge) aluminium cable, with admissible current and low-frequency resistance equal to, respectively, $I_{a d m}=328 \mathrm{~A}$ and $D C R$ (direct current resistance) $=$ $0.3922 \Omega / \mathrm{km}$;

- branches: $1 / 0$ AWG aluminium cable, with $I_{\text {adm }}=$ $244 \mathrm{~A}$ and $R C R=0.6047 \Omega / \mathrm{km}$.

The RGR-104 feeder is characterized by the presence of multigrounded neutral at every $300 \mathrm{~m}$ and at every pole where there is distribution equipment installed. The simulations to evaluate the feeder's performance were employed through calculations of power flow with the software Interplan, using as input, the RGR-104's georeferenced data and phase and neutral current measurements at the substation. The methodology developed in order to calculate the power flow allows, as well as other parameters, the phase and

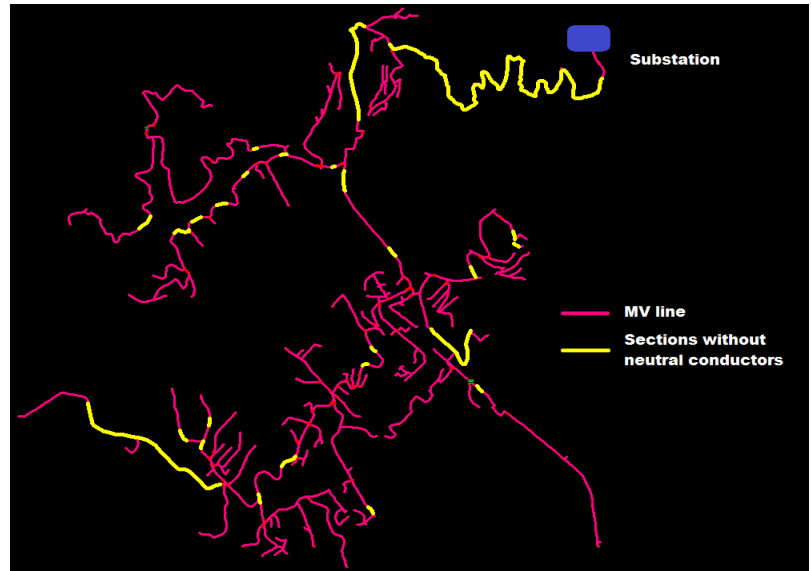

Fig. 1 RGR-104 single-line diagram with indications of the sections without neutral conductors.

Table 1 Measured and simulated values of phase and neutral currents at the substation of RGR-104.

\begin{tabular}{lll}
\hline & Measured values & Simulated values \\
\hline$I_{D}(\mathrm{~A})$ & 445 & 437 \\
$I_{E}(\mathrm{~A})$ & 452 & 424 \\
$I_{F}(\mathrm{~A})$ & 417 & 473 \\
$I_{N}(\mathrm{~A})$ & 32 & 37 \\
\hline
\end{tabular}

neutral current, neutral-ground voltage and electrical losses to be simulated.

Based on the georeferenced data and phase and neutral currents measurements, adjustments were performed in order to obtain current and imbalance values close to the data, as shown in Table 1.

The ground resistivity and the contributions of the ground resistances at the consumers' entrance and adjacent feeders are not considered in the simulations. Therefore, unless otherwise indicated, the $R g$ was assumed to be $25 \Omega$ for poles that are grounded. The $R g$ refers to the resulting value of a grounding isolated system, in other words, not connected to the multigrounded network.

\section{Results and Discussion}

To evaluate the impacts of the neutral conductor's absence at specific sections over the performance and safety of RGR-104 power distribution network, a methodology of power flow based on Gauss elimination method was developed in Ref. [4] and simulations were made with the software Interplan. 
According to Ref. [2], the maximum Vng (neutral-to-ground voltage) level must not overcome $10 \mathrm{~V}$ in normal operating conditions.

Initially, the performance and safety of RGR-104 MV line were evaluated without taking into account the neutral conductor theft (reference case). Afterwards, its performance was evaluated considering the real conditions, and solutions were proposed in order to mitigate the damages due to the neutral conductor's absence.

\subsection{Reference Condition of RGR-104 Distribution Network}

Fig. 2 shows the performance of RGR-104 MV line - in terms of safety levels - for the reference case. Red areas are classified as critical and refer to poles with $V n g>10 \mathrm{~V}$, yellow areas indicate poles with $5 \mathrm{~V}$ $<V n g<10 \mathrm{~V}$, and green areas are the poles with $V n g<$ $5 \mathrm{~V}$.

The results show that, even for the reference case, part of the MV line is within the acceptable limits (yellow areas, $5 \mathrm{~V}<V n g<10 \mathrm{~V}$ ) and some are already critical areas. In this case, about $7 \%$ of the 2,975 poles of RGR-104 feeder have $V n g>10 \mathrm{~V}$, considering the maximum value of $V n g$ and technical losses are, respectively, $21 \mathrm{~V}$ and $360 \mathrm{~kW}$. The critical areas in the reference case are due to the imbalance created by the sections with delta transformers (single-phase). The critical area density grows following the $R g$ growth.

In the following analysis, the performance of RGR-104 MV line is evaluated for the real condition, i.e., taking into account the sections with the lack of neutral conductor, and solutions are presented to mitigate the damages due to neutral conductor's theft.

\subsection{Real Condition of RGR-104 Distribution Network}

The calculations forth target the evaluation of the system's performance, concerning the safety levels, taking into account the sections without neutral conductors. According to the AES Eletropaulo, 20\% of the $51 \mathrm{~km}$ length of RGR-104 distribution network lack

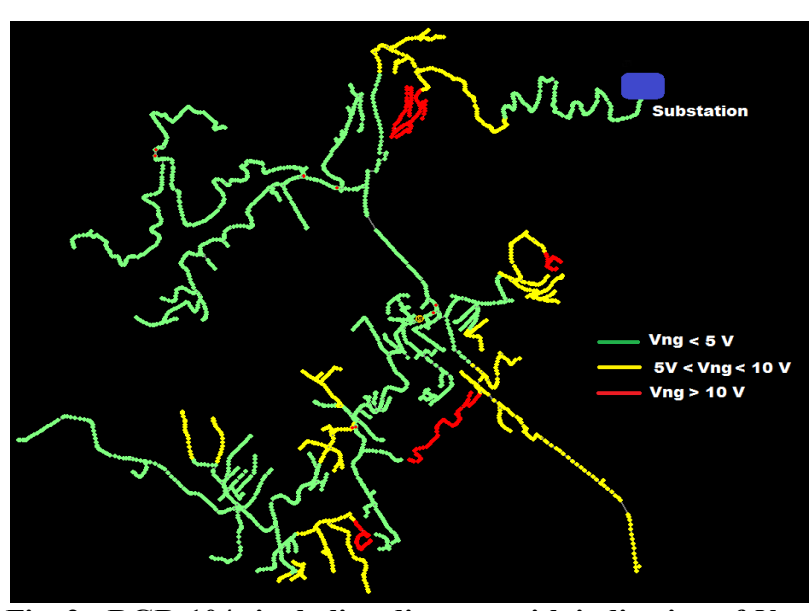

Fig. 2 RGR-104 single-line diagram with indication of $\mathrm{Vng}$ levels, for the reference condition of RGR-104 distribution network. $R g=25 \Omega$.

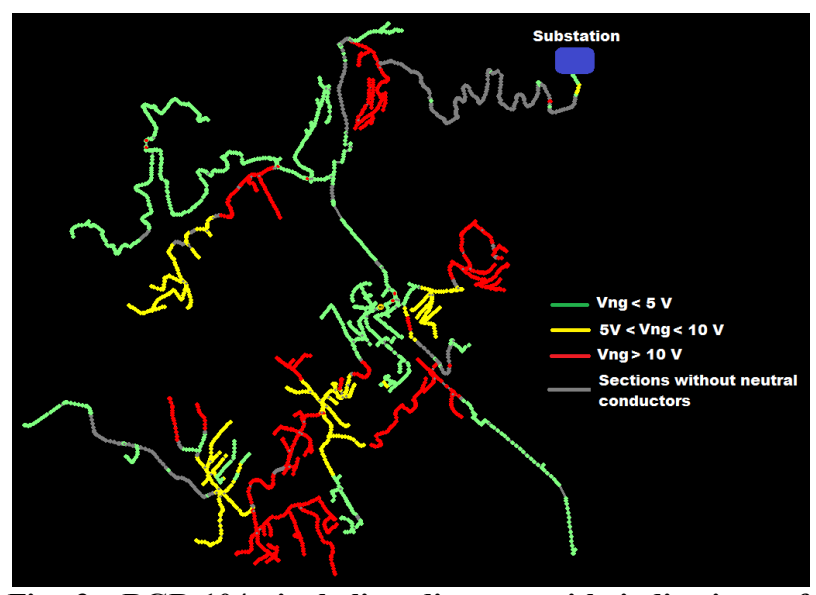

Fig. 3 RGR-104 single-line diagram with indications of $V n g$ levels, for the real condition of RGR-104 distribution network. $R g=25 \Omega$.

neutral conductors, as shown by the single-line diagram in Fig. 1.

Fig. 3 presents the $V n g$ levels for the real operation condition of RGR-104 feeder.

The results show that the absence of the neutral conductor at specific sections of power distribution lines worsens the operational condition, increases the number of poles with $V n g>10 \mathrm{~V}$ and the maximum neutral-to-ground voltage. In this case, $30 \%$ of the 2,975 poles of RGR-104 distribution network have $V n g>10 \mathrm{~V}$, being $51 \mathrm{~V}$ - the maximum value of $V n g$.

In regard to the technical losses, it is clear that the lack of neutral conductors increases the technical loss to $368 \mathrm{~kW}$. 


\subsection{Alternative Solution to Mitigate the Damages due} to the Neutral Conductor Absence and Improve the Feeder's Safety Condition

To improve the technical performance of RGR-104 distribution network showed in Fig. 3, an evolutionary algorithm was developed and implemented on the software Interplan capable of evaluating the performance of the feeder, and indicating suitable ground resistance values at specific grounding points along the distribution network to keep the system performance at satisfactory technical and safety levels [5].

The solution to eliminate the aluminium cable theft was the use of bimetallic conductors in the MV line sections without the neutral conductors, as shown in Fig. 1 [6]. According to Ref. [4], the branches were installed with bimetallic steel/aluminium wire 7N9 53\% IACS (International Annealed Copper Standard) instead of 1/0 AWG aluminium cable and, in the main feeder, bimetallic steel/aluminium wire 7N7 53\% IACS instead of 3/0 AWG aluminium cable.

The use of bimetallic wire at the sections with neutral conductor theft reduces the Vng levels and technical losses to values close to the ones found in reference case (Fig. 2), as shown in Fig. 4.

According to the results of Interplan, in order to keep the safety conditions of RGR-104 within the acceptable levels [2] and, consequently, reduce the technical losses, it is important to use the bimetallic conductors in all the sections with neutral absence and reduce the ground resistance values of the grounding points indicated in Fig. 4 to about $5 \Omega$.

Fig. 5 presents the single-line diagram of RGR-104 MV with indications of neutral-to-ground levels for the case of bimetallic conductors usage at the sections where the neutral conductors were stolen (Fig. 1) and $R g=5 \Omega$ in the grounding points indicated in Fig. 4.

Comparing Figs. 3 and 5, it is clear that the proposed solution has significantly improved the RGR-104 voltage profile, keeping the $V n g$ voltage within the limit of $10 \mathrm{~V}$. In this case, there was an important

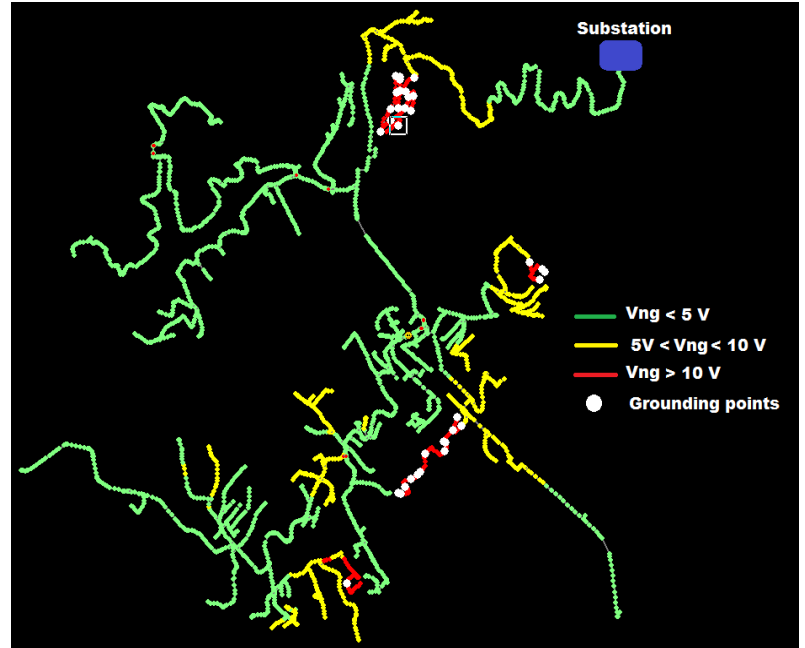

Fig. 4 RGR-104 single-line diagram with indications of Vng levels and grounding points of the critical areas, for the case of using bimetallic conductors at the sections where the neutral conductors were stolen $(R g=25 \Omega)$.

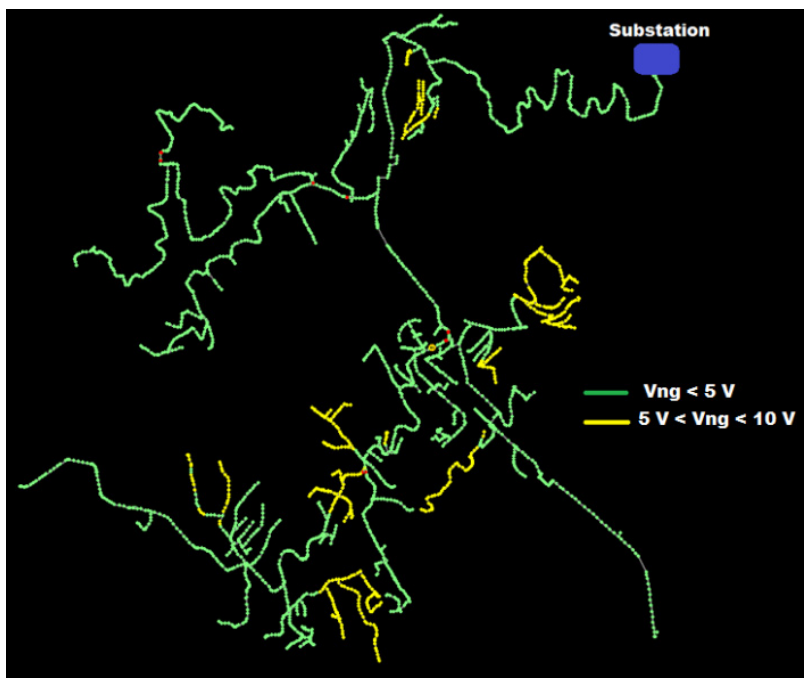

Fig. 5 RGR-104 single-line diagram with indications of $V n g$ levels, for the case of using bimetallic conductors at the sections where the neutral conductors were stolen and $R \boldsymbol{g}=$ $5 \Omega$ in the grounding points indicated in Fig. 4.

reduction in the technical losses $(2.5 \%)$ in comparison to the real case (Fig. 3).

\section{Conclusions}

The paper evaluates the impact of neutral conductor absence at specific sections over the performance of power distribution lines, and has proposed technical solution to mitigate the damages caused by the lack of neutral conductor and improve the distribution system's safety condition. The simulations were 
performed to the RGR-104 feeder due to the high theft percentage and the results were employed in the field.

To evaluate the system's performance taking into account the neutral conductor absence at specific sections of the distribution line, a new methodology of power flow based on Gauss elimination method was developed and implemented on the software Interplan. The results of the simulations have shown that the absence of neutral conductor increases the Vng, which diminishes the system's safety.

To improve the safety levels of RGR-104, an evolutionary algorithm was developed and implemented on the software Interplan capable of indicating suitable ground resistance values at specific grounding point along the distribution network to keep the system's performance at satisfactory technical and safety levels. Some alternative solutions to improve the grounding systems and, consequently, reduce the ground resistance, were proposed in Refs. [4, 7-10].

As an alternative to eliminate the frequent thefts of aluminum cables, bimetallic conductors were used as replacement in the sections.

According to the results presented in Fig. 2 (reference case), the imbalance regarding the delta transformers alongside the distribution line causes the increase to the neutral-to-ground voltage levels, overcoming, in some areas, the limit values in Ref. [2].

Furthermore, it is important to mention that other alternatives may be used in case that the voltage levels overcome the limit values in Refs. [4, 7-10], such as:

- changing the conductor's type;

- balancing the loads through the primary distribution line;

- gradual conversion of the wye to delta connection transformers (Fig. 6).

Additionally, preventive measures can be taken, such as the use of DAT (transversal distribution network) system in the regions with high incidence of neutral conductors' theft.

It should be noted that the results presented in this paper may be used as reference for planning and

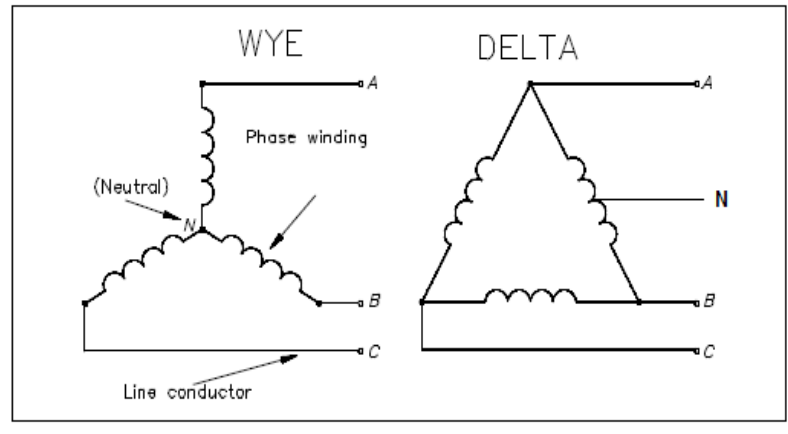

30 AC Power Connections

Fig. 6 Example of wye to delta connection transformer.

performance improvements in distribution networks. Nevertheless, in practice, the influence of the consumer's and other nearby feeder's groundings, ground resistance and soil resistivity must be taken into account.

\section{References}

[1] Zipse, D. W. 2003. "The Hazardous Multigrounded Neutral Distribution System and Dangerous Stray Currents." Presented at the IEEE Petroleum and Chemical Industry Conference, Houston, USA.

[2] AES Eletropaulo. 1993. Grounding: Technical Instructions for the Distribution of Energy (ID-5.50). Technical report for AES Eletropaulo Distribution Utility, Sao Paulo, Brazil.

[3] Oliveira, C. C. B., Kagan, N., Guaraldo, J. C., El Hage, F. S., Meffe, A., and Filho, M. M. 2004. "Interplan-A Tool for Planning High, Medium and Low Voltage Networks." Presented at the IEEE Transmission and Distribution Conference and Exposition, Denver, USA.

[4] Antunes, A. U., Meffe, A., Takahata, D., Romero, F., Nanni, M., and Lange, F. L. 2010. Specifications of Power Flow Methodology and Technical Economic Analysis of Alternative Solutions to Mitigate Damages due to Neutral Conductors Theft. Technical report for AES Eletropaulo Distribution Utility, Sao Paulo, Brazil.

[5] Antunes, U., Meffe, A., Takahata, D., Kamikoga, D. H., Romero, F., and Lange, F. L. 2010. Technical Solutions to Mitigate Problems due to Neutral Conductors Theft in AES Eletropaulo MV Power Distribution System. Technical report for AES Eletropaulo Distribution Utility, Sao Paulo, Brazil.

[6] Cipoli, J. A., Zamboni, L., Borelli, A. B., Marco, M. A., and Nogueira, E. D. B. 2005. "Usage of Bimetallic Conductors in MV and LV Lines of Low-Income Community." Presented at III CITENEL Brazilian Congress of Technological Innovation in Electricity, 

Theft in MV (Medium Voltage) Power Distribution Systems

Florianópolis, Brazil.

[7] Bezerra, S. R. C., and Kanashiro, A. G. 2010. "Grounding System with Concrete-Encased Grounding Electrodes." Presented at III SBSE Brazilian Symposium on Electric Systems, Ilha Solteira, Brazil.

[8] Fagan, E. J., and Lee, R. H. 2009. "The Use of Concrete-Enclosed Reinforcing Rods as Grounding Electrodes." IEEE Transactions on Industry and General Applications IGA-6 (4): 337-48.
[9] Filho, F. G. S. 2006. "Electric Power Supply Distribution Network through DAT (Transversal Distribution Network) System.” CIER (Comissión de Integración Energética Regional).

[10] Souza, K. T., Moura, R. F., and Cintra, G. 2007. "Study of Alternatives for Grounding Construction in High Resistivity Ground.” Presented at II Brazilian North and Northeast Congress on Research and Development in Technology Education, Brazil. 